\title{
High Prevalence of Myopia and Low Hyperopia Reserve in 4411 Chinese Primary School Students and Associated Risk Factors
}

\section{Yu Yue}

First Affiliated Hospital of Chongqing Medical University, Chongqing Eye Institute

Xianmao Liu

First Affiliated Hospital of Chongqing Medical University, Chongqing Eye Institute

Shu Yi

Chongqing General Hospital

Bo Liu

Southwest Hospital, Southwest Eye Hospital, Third Military Medical University

\section{Hong Yi}

Chongqing General Hospital

Hong Li ( $\sim$ lihongcqmu@163.com )

First Affiliated Hospital of Chongqing Medical University, Chongqing Eye Institute

\section{Research Article}

Keywords: Myopia, Prevalence, Ocular biometrics, Risk factors

Posted Date: October 22nd, 2021

DOI: https://doi.org/10.21203/rs.3.rs-962786/v1

License: (1) This work is licensed under a Creative Commons Attribution 4.0 International License. Read Full License 


\section{Abstract}

Purpose: To investigate the prevalence of myopia in Chinese primary school students and their ocular biometrics including axial length (AL), corneal radius of curvature (CRC) and spherical equivalent refraction (SER). To analyze their association with potential myopia risk factors, such as body mass index (BMI), cram school, time of outdoor activity and electronic screen use.

Methods: In this cross-sectional study of 4500 primary school students from 5 schools, participants underwent refraction using non-cycloplegic autorefractor and visual acuity testing. Myopia was defined as SER $\leq-0.50$ diopter (D) and uncorrected visual acuity (UCVA) $\nabla 0.00 \log M A R(6 / 6)$. Logistic regression models were used to determine factors associated with myopia.

Results: After excluding 389 participants, the overall prevalence of myopia was $33.6 \%$. The prevalence of high myopia was $0.6 \%$. The prevalence of myopia in girls was significantly higher than that in boys $\otimes 37.6 \%$ vs. $30.0 \%, p<0.001)$. The height, weight and BMI were significantly associated with $A L(r=0.471$, $r=0.440, r=0.276, p<0.001$, respectively). AL/CRC ratio was more highly correlated with SER than $A L$ alone. Age $(p<0.001)$, parental myopia $(p=0.001)$ and lack of outdoor activity between classes $(p=$ 0.049 ) were independently associated with higher prevalence rates of myopia.

Conclusion: The prevalence of myopia among Chinese schoolchildren is alarming high. Consistent with previous cross-sectional data, AL/CRC could function as myopia detection indicator. The hyperopia reserve among children aged between $6 \sim 7$ years was low. Healthcare providers need to raise parents' awareness of the importance of regular eye examination and proper optical correction.

\section{Introduction}

Myopia, the most common refractive error, is the main cause of presenting visual impairment worldwide. The prevalence of myopia has been observed to be increasing at a dramatic rate in young East Asians. In children aged 6-8 years, $18.0 \%$ 34.7\% were myopic in developed areas including Beijing, ${ }^{1}$ Hongkong ${ }^{2}$ and Singapore. ${ }^{3}$ According to a recent meta-analysis summarizing the prevalence of myopia in 7 to 12 year-old children in China, the myopia prevalence has increased from $25.3-32.8 \%$ before as compared to after $2008 .{ }^{4}$

Although the refractive error can be corrected optically, myopia has been associated with complications such as retinal detachment, myopic macular degeneration and choroidal neovascularization. ${ }^{5,6}$ These complications can cause irreversible visual impairment and considerable visual morbidity. Among Chinese university students, studies reported that $86.8 \%$ had myopia in Nanjing, ${ }^{7} 94.9 \%$ had myopia and $19.5 \%$ had high myopia in Shanghai. ${ }^{8}$ The high prevalence of myopia and high myopia in Chinese students has strong impact on their quality of life and inevitably increases the socioeconomic burden. Therefore, it is of prime importance to identify primary school students at a higher risk of pathological 
myopia development in order to start close ophthalmic surveillance and control myopia progression with appropriate measures.

Currently, researchers often evaluate the refractive status in large cohorts of school children by refraction without cycloplegia, and by measuring axial length $(A L)$, corneal radius of curvature $(C R C)$ and spherical equivalent refraction (SER). However, assessing the likelihood of future myopia development is still difficult. Scheiman et al reported that the ratio of $A L$ to $C R C(A L / C R C)$ could be a better marker of myopia progression than $\mathrm{AL}$ alone. ${ }^{9}$ Hyperopia reserve is defined as the physiological hyperopia during infancy and young childhood, which will decrease during emmetropization. ${ }^{10}$ It is estimated that the lack of hyperopia reserve might be a predictor of future myopia formation.

The mechanism of myopia formation and progression still remains uncertain. Genetic contribution to refractive error is important nevertheless it could not explain the recent considerable increase in myopia prevalence. Based on modern epidemiological and genetic analysis, it is generally agreed that the formation of school myopia is a complex developmental process susceptible to environmental modulation. ${ }^{11}$ Environmental factors such as educational pressures and reductions of time outdoors were causal factors of myopia. ${ }^{12}$ Nowadays, the electronic screen use, ${ }^{13}$ the body mass index (BMI) were also reported to be potential myopia risk factors. ${ }^{14}$ Hence finding the major causal factors and ameliorating children's studying condition and daily behavior could be the key to myopia control.

In this study, we aimed to determine rates of incidence of myopia in primary school students in Chongqing, the largest city in China. And we aimed to analyze the refractive characteristics and their association with potential myopia risk factors, such as BMI, time of cram school, electronic screen use, etc. We also conducted the evaluation of the ratio of $A L$ to $C R C$, the hyperopia reserve as potential marker or predictor of myopia formation in this population.

\section{Methods}

\section{Study design and population}

In 2021, we conducted a cross-sectional survey of myopia prevalence in children from 5 primary schools in Chongqing, China. Three schools are located in urban area and two schools are from less developed towns. Every student from each grade of the 5 primary schools was invited in this survey. The purpose and procedures of the study were explained to all of them. A written consent form was signed by the parents. All of the study protocols conformed to the Tenets of the Declaration of Helsinki and the research protocols were approved by the Committee of Ethics in Chongqing People's Hospital. Ethical approval number: KYS2021-011-01. Students who had systemic pathologies were excluded.

\section{Ocular examination}

Visual acuity was measured as uncorrected visual acuity (UCVA) (using a logarithmic VA chart) at a distance of 5 meters. Refraction was measured three times in each eye in a darkened room without 
cycloplegia with the autorefractometer KR-800 (Topcon, Japan). Axial length, CRC and central corneal thickness were measured with the AL-Scan (NIDEK Ltd., Tokyo, Japan). Axial length was defined as the distance between the tear film and the retinal pigment epithelium. All the data were uploaded in an online

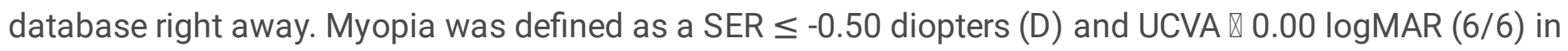
either eye. High myopia was defined as a SER $\leq-6.00 \mathrm{D}$. Hyperopia was categorized by the degree of refractive error. Low hyperopia was defined as a SER $\leq+2.00 \mathrm{D}$. Moderate to high hyperopia was defined as a SER $\geq+2.25 \mathrm{D}$. After excluding subjects with moderate to high hyperopia, an adequate hyperopia reserve was defined as SER $\geq+1.00 D$ for $6-8$ years old, $\geq+0.75 D$ for 9 years old, $\geq+0.50 D$ for 10 years old, $\geq+0.25 \mathrm{D}$ for 11 years old and $\geq 0 \mathrm{D}$ for 12 years old according to the handbook for prevention and control of myopia in children and adolescents by the National Health Commission of the People's Republic of China. ${ }^{15}$

As for quality control, $5 \%$ of participants were randomly chosen from the database at the end of day during the fieldwork. The UCVA and auto-refraction were retested. Data with an error rate lower than $5 \%$ was accepted as valid.

\section{BMI measurements}

Height and weight were measured at the same time by SH-200 height-weight measurement body scale meter (Shanghe Ltd., Zhengzhou, China) with the subject standing barefoot on the base, without shoes and heavy coats. BMI was calculated as weight/height and recorded in kilograms per square meter $\left(\mathrm{kg} / \mathrm{m}^{2}\right)$.

\section{Questionnaire}

A questionnaire was developed by two ophthalmologists from Department of Ophthalmology, Chongqing General Hospital and answered by the parents of our subjects. The questionnaire included questions about the educational attainment of parents, parental myopia, electronic screen use, cram school, reading posture and duration of outdoor activities (See Supplementary Material 1 and 2).

\section{Statistical analysis}

All statistical analyses were performed with the SPSS statistics 20 for Windows (SPSS Inc., IBM, Somers, New York, USA). AL, SER, ACD, CRC and AL/CRC were not distributed normally based on Shapiro-Wilk test. Thus, the significance of the biometric differences between different grades (6 grades in total) were calculated by Kruskal-Wallis test. The difference of myopia prevalence between different genders were calculated by Chi square test. The association between height, weight and BMI and AL were analyzed by Spearman's correlation analyses. Univariate analysis was performed to evaluate potential associations. These included age, sex, BMI, parental myopia, outdoor activity during break between classes, time of PE lessons, time of cram school, electronic screen use. Afterwards, multivariate logistic regression modeling was performed to analyze all statistically significant factors found in the univariate analysis. $P$ values are 2 sided and $\mathrm{P}<0.05$ was considered statistically significant. 


\section{Results}

\section{Demographic characteristics}

4500 participants were recruited in the initial research. A total of 4411 participants were available for the statistical analysis after excluding 88 subjects with missing data, 1 subject diagnosed with Down syndrome. Table 1 presents the demographic characteristics of the analysis cohort by myopic or nonmyopic. The response rate of the questionnaire was 749 out of 4411 . One hundred and thirty-two subjects chose "unknown" in the questionnaire and were excluded during analysis.

Table 1 Demographic characteristics

\begin{tabular}{|c|c|c|c|c|}
\hline Variables & Myopic & Non-myopic & Total & $P$ value \\
\hline Age (years) & $10(8,11)$ & $8(7,10)$ & $9(7,10)$ & $₫ 0.001^{\mathrm{a}}$ \\
\hline \multirow[t]{2}{*}{ Height (cm) } & 141.0 & 132.0 & 135.0 & \multirow[t]{2}{*}{$\nabla 0.001^{\mathrm{a}}$} \\
\hline & $(133.0,149.0)$ & $(125.0,141.0)$ & $(127.0,144.5)$ & \\
\hline \multirow[t]{2}{*}{ Weight (kg) } & 36.1 & 29.9 & 31.8 & \multirow[t]{2}{*}{$\nabla 0.001^{a}$} \\
\hline & $(29.7,44.4)$ & $(25.3,37.0)$ & $(26.4,40.0)$ & \\
\hline \multirow[t]{2}{*}{ BMI $\left(\mathrm{kg} / \mathrm{m}^{2}\right)$} & 17.95 & 17.07 & 17.36 & \multirow[t]{2}{*}{$\llbracket 0.001^{\mathrm{a}}$} \\
\hline & $(16.43,20.36)$ & $(15.81,18.94)$ & $(15.99,19.46)$ & \\
\hline Gender & & & & $\varangle 0.001^{b}$ \\
\hline Male & $684 \rrbracket 30.0 \% \rrbracket$ & $1598 \rrbracket 70.0 \% \rrbracket$ & 2282 & \\
\hline female & $800 \rrbracket 37.6 \% \rrbracket$ & $1329 \rrbracket 62.4 \% \rrbracket$ & 2129 & \\
\hline Wearing spectacles & & & & $\varangle 0.001^{b}$ \\
\hline Yes & $377 \rrbracket 80.9 \% \rrbracket$ & 89®19.1\%】 & 466 & \\
\hline No & 1107ه28.1\%区 & $2838 ه 71.9 \% \rrbracket$ & 3945 & \\
\hline Total & 1484 (33.6\%) & $2927 \rrbracket 66.4 \% \rrbracket$ & 4411 & \\
\hline
\end{tabular}

a. Mann-Whitney U test b. Chi-square test

Data are presented as medians and quartiles $(p 25, p 75)$ or as $n(\%)$.

\section{Prevalence of myopia}

The overall prevalence of myopia was $33.6 \%$ (1484 of 4411$)$. The prevalence of high myopia was $0.6 \%$ (27 of 4411). The prevalence rate of myopia in different grades and sexes was shown in Table 2 . The 
incidence of myopia increased significantly as the grade levels rose $\left(x^{2}=522.169, p<0.001\right)$, from $8.6 \%$ in first grade to $55.6 \%$ in sixth grade. After adjusted by Bonferroni method, significant difference was found between every two grades from $1^{\text {st }}$ to $4^{\text {th }}$ grade, and between $4^{\text {th }}$ and $6^{\text {th }}$ grade. There was no significant difference in the incidence of myopia between $4^{\text {th }}$ and $5^{\text {th }}$ grade, $5^{\text {th }}$ and $6^{\text {th }}$ grade. The prevalence of myopia in girls was significantly higher than that in boys $₫ 37.6 \%$ vs. $30.0 \%, x^{2}=28.517, p<0.001$ ).

Table 2. Prevalence rate of myopia in different grades and sexes

\begin{tabular}{|c|c|c|c|c|c|c|c|c|}
\hline \multirow[t]{2}{*}{ Grade } & \multicolumn{2}{|l|}{ Male } & \multicolumn{2}{|l|}{ Female } & \multirow{2}{*}{$\begin{array}{l}\text { Difference } \\
\text { between sexes }\end{array}$} & \multirow{2}{*}{$\begin{array}{l}\text { Total } \\
\text { (\%) }\end{array}$} & \multirow{2}{*}{$\begin{array}{l}\text { Increase } \\
\text { rate } \\
\%\end{array}$} & \multirow[t]{2}{*}{$P$ value } \\
\hline & Myopic & $\%$ & Myopic & $\%$ & & & & \\
\hline $1^{\text {st }}$ & 42 & 5.1 & 28 & 3.4 & $-1.7 \%$ & 817 & & \\
\hline $2^{\text {nd }}$ & 78 & 9.6 & 96 & 11.8 & $2.2 \%$ & 812 & 12.9 & $\begin{array}{l}x^{2}=52.891, p \\
0.001^{a}\end{array}$ \\
\hline $3^{\text {rd }}$ & 141 & 17.2 & 127 & 15.5 & $-1.7 \%$ & 819 & 11.3 & $\begin{array}{l}x^{2}=26.327, p \\
0.001^{a}\end{array}$ \\
\hline $4^{\text {th }}$ & 137 & 19.4 & 180 & 25.5 & $6.1 \%$ & 705 & 12.2 & $\begin{array}{l}x^{2}=24.006, p \\
0.001^{a}\end{array}$ \\
\hline $5^{\text {th }}$ & 148 & 21.2 & 196 & 28.0 & $6.9 \%$ & 699 & 4.2 & $\begin{array}{l}x^{2}=2.543 \\
p=0.111^{a}\end{array}$ \\
\hline $6^{\text {th }}$ & 138 & 24.7 & 173 & 30.9 & $6.3 \%$ & 559 & 6.4 & $\begin{array}{l}x^{2}=5.133 \\
p=0.023^{a}\end{array}$ \\
\hline Total & 684 & 15.5 & 800 & 18.1 & & 4411 & & \\
\hline
\end{tabular}

a. Chi-square test, difference between grades

\section{Refraction and ocular biometric parameters}

The mean SERs were 0.31 D, -0.27 D, -0.58 D, -0.63D, -0.83D and - 1.15 for participants from $1^{\text {st }}$ to $6^{\text {th }}$ grade respectively (Figure 1). Significant differences ( $p \varangle 0.01$ ) were found between each two grades, except between $4^{\text {th }}$ and $5^{\text {th }}$ grade $(p=0.117)$. Spearman's correlation test showed that the AL and AL/CRC were closely associated with SER(AL: $r=-0.531$, p $₫ 0.001$; AL/CRC: $r=-0.639$, $p \otimes 0.001)$.

The mean ALs were $22.60,23.11,23.54,23.56,23.63$ and $23.92 \mathrm{~mm}$ from $1^{\text {st }}$ to $6^{\text {th }}$ grade respectively. Significant differences ( $\mathrm{p} \otimes 0.01)$ were found between each two grades, except between $3^{\text {rd }}$ and $4^{\text {th }}(p=$ $0.060), 5^{\text {th }}$ and $6^{\text {th }}$ grade $(p=0.268)$. The mean ratio of $A L$ to $C R C$ were $2.89,2.96,2.99,3.01,3.03,3.05$ from $1^{\text {st }}$ to $6^{\text {th }}$ grade respectively. Significant differences $(p \otimes 0.01)$ were found between each two grades, 
except between $5^{\text {th }}$ and $6^{\text {th }}$ grade $(p=0.931)$. The refractive characteristics of the myopic and nonmyopic groups were shown in Table 3.

Table 3. Refractive characteristics

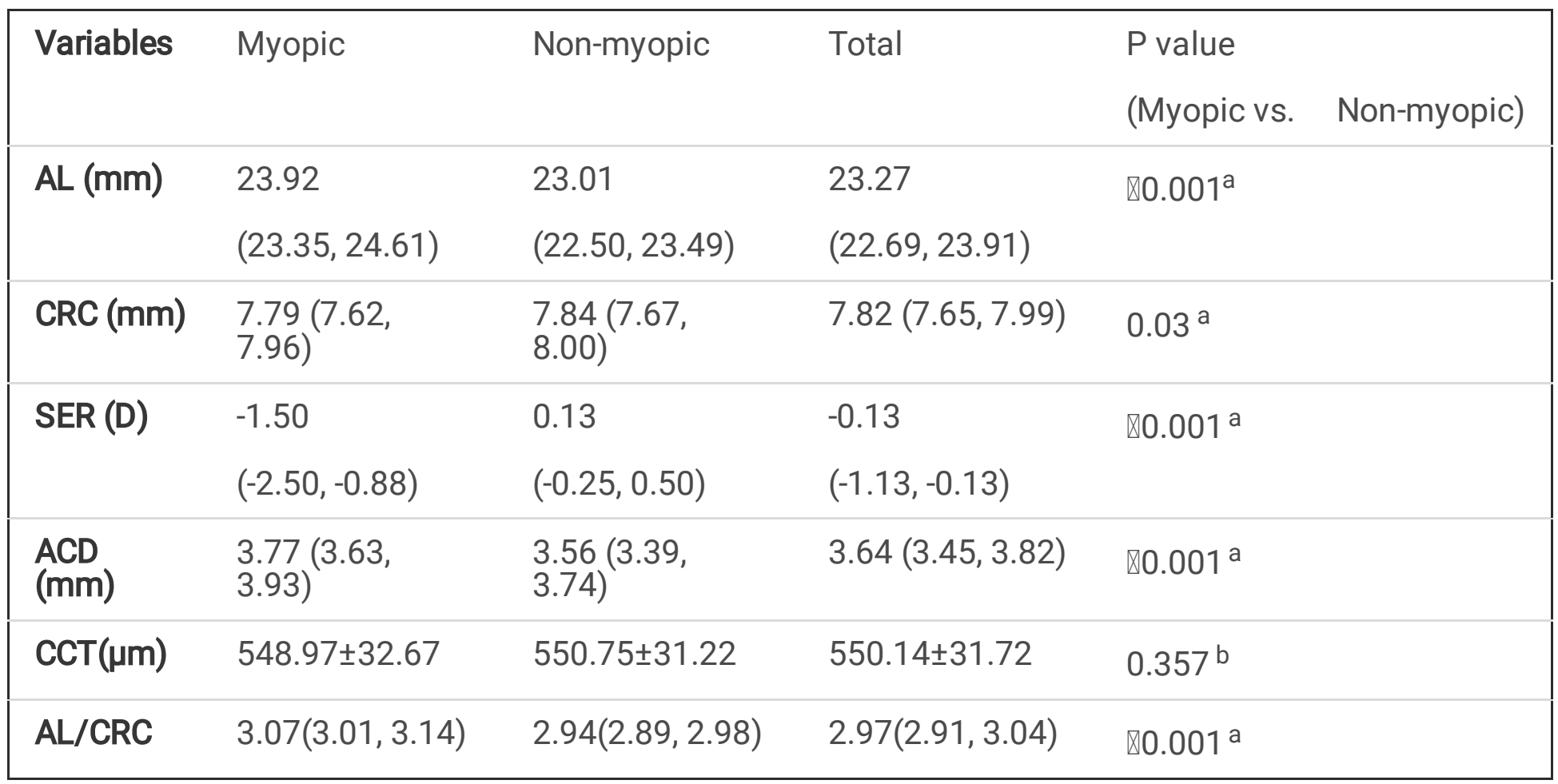

Data are presented as medians and quartiles $(p 25, p 75)$ or as the means with SD

a. Mann-Whitney U test

b. Independent t-test

There were 384 participants (8.7\%) out of 4411 with adequate hyperopia reserve in total. The prevalence of moderate and hyperopia was $0.8 \%(36 / 4411)$. As is shown in Figure 2 , the percentage of subjects with adequate hyperopia reserve was low, especially in younger participants. Subjects with age over 12 years old were not included in this figure.

\section{Relationship between body stature and refractive characteristics}

The height, weight and BMI were found to be significantly associated with $A L(r=0.471, p<0.001 ; r=0.440$, $p<0.001 ; r=0.276, p<0.001$, respectively), SER ( $r=-0.376, p<0.001 ; r=-0.334, p<0.001 ; r=-0.189, p<0.001$, respectively) and $A L / C R C(r=0.445, p<0.001 ; r=0.406, p<0.001 ; r=0.240, p<0.001$, respectively).

\section{Multiple logistic regression analysis}

Univariate analysis showed that educational attainment of parents, time of homework, near visual work, reading and writing posture, time of outdoor activities were not related with incidence of myopia (results shown in Supplementary material 3). Relevant risk factors validated by univariate analysis were listed in 
Table 4. Multiple logistic regression analysis was then performed. Results showed that parental myopia was associated with a greater prevalence of myopia (Table 4).

Table 4. Analysis of risk factors of myopia by multiple logistic regression 


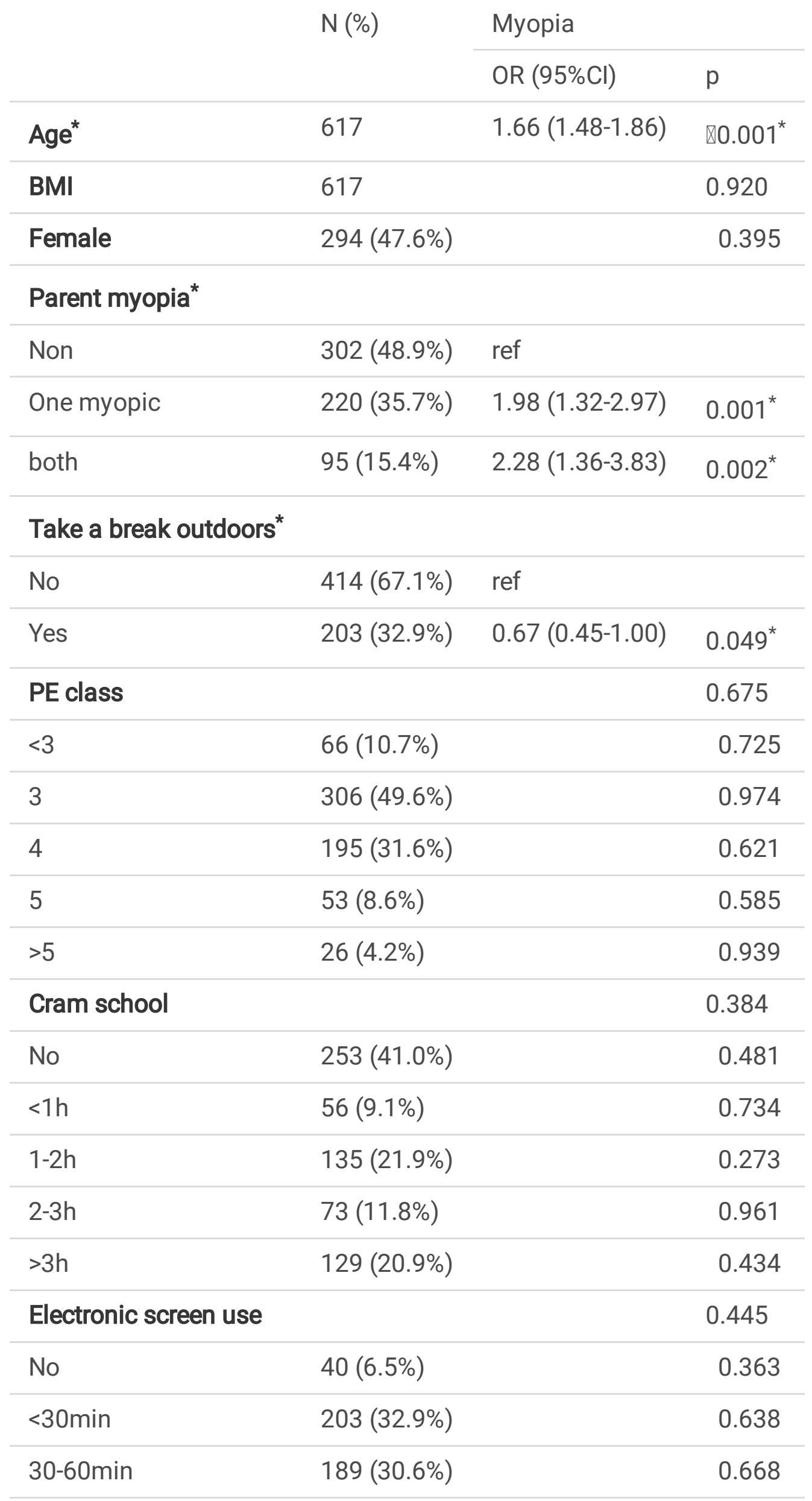




\begin{tabular}{lll}
\hline $1-2 \mathrm{~h}$ & $118(19.1 \%)$ & 0.390 \\
\hline $2-3 \mathrm{~h}$ & $38(6.2 \%)$ & 0.094 \\
\hline$>3 \mathrm{~h}$ & $58(9.4 \%)$ & 0.492
\end{tabular}

Data are presented as $\mathrm{n}(\%)$.

* statistically significant

Other independently associated factors for myopia prevalence were age $(p<0.001)$ and lack of outdoor activity during break between classes $(p=0.049)$. Parental myopia was also associated with longer axial length (regression coefficient 0.100 [95\% $\mathrm{Cl}, 0.026-0.174], \mathrm{p}=0.008$ ) and greater $\mathrm{AL} / \mathrm{CRC}$ (regression coefficient 0.012[95\% Cl, 0.004-0.020], $\mathrm{p}=0.005$ ) (Table 5).

Multiple logistic regression analysis showed that older age, the female sex, higher BMI, more cram school classes were independently associated with longer axial length. Factors which independently associated with greater $\mathrm{AL} / \mathrm{CRC}$ were similar, except BMI.

Table 5. Analysis of association between risk factors and AL and AL/CRC by multiple linear regression

\begin{tabular}{|c|c|c|c|c|}
\hline & \multicolumn{2}{|l|}{$\mathrm{AL}$} & \multicolumn{2}{|l|}{$\mathrm{AL} / \mathrm{CRC}$} \\
\hline & $\begin{array}{l}\text { regression coefficient, } 95 \% \\
\mathrm{Cl}\end{array}$ & $\begin{array}{l}\mathrm{p} \\
\text { value }\end{array}$ & $\begin{array}{l}\text { regression coefficient, 95\% } \\
\mathrm{Cl}\end{array}$ & $\begin{array}{l}\mathrm{p} \\
\text { value }\end{array}$ \\
\hline $\mathrm{Age}^{*}$ & $0.266[0.224-0.308]$ & $0.001^{*}$ & $0.029[0.025-0.034]$ & $0.001^{*}$ \\
\hline $\mathrm{BMI}^{*}$ & $0.024[0.001-0.047]$ & $0.044^{*}$ & $0.002[0.000-0.005]$ & 0.102 \\
\hline Female $^{*}$ & $0.582[0.453-0.710]$ & $0.001^{*}$ & $0.021[0.007-0.036]$ & $0.004^{*}$ \\
\hline Parental myopia* & $0.100[0.026-0.174]$ & $0.008^{*}$ & $0.012[0.004-0.020]$ & $0.005^{*}$ \\
\hline $\begin{array}{l}\text { Take a break } \\
\text { outdoors }\end{array}$ & $-0.090[-0.225-0.046]$ & 0.194 & $-0.008[-0.023-0.008]$ & 0.318 \\
\hline PE class & $0.016[-0.054-0.085]$ & 0.662 & $0.005[-0.003-0.013]$ & 0.229 \\
\hline Cram school $^{\star}$ & $0.045[0.002-0.089]$ & $0.042^{*}$ & $0.005[0.000-0.010]$ & $0.047^{*}$ \\
\hline $\begin{array}{l}\text { Electronic screen } \\
\text { use }\end{array}$ & $-0.010[-0.060-0.039]$ & 0.682 & $0.000[-0.006-0.006]$ & 0.985 \\
\hline
\end{tabular}




\section{Discussion}

In this study, we investigated the prevalence of myopia, the refractive characteristics and their associated risk factors among primary school children in Chongqing. The overall prevalence of myopia among primary school children in Chongqing was $33.6 \%$. The prevalence of high myopia was $0.6 \%$. The prevalence of myopia rose dramatically from $8.6 \%$ in the 1 st grade to $55.6 \%$ in the 6 th grade. Compared with boys, more girls were myopic (37.6\% vs. $30.0 \%$ ). A high correlation between AL/CRC and SER was found, and its coefficient of correlation was higher than that between AL and SER ( $r=-0.639$ for AL/CRC vs. $r=-0.531$ for $A L$ ). We investigated the hyperopia reserve among our subjects and found only $8.7 \%$ with adequate hyperopia reserve. Age, lack of outdoor activity during break between classes and parental myopia were identified as independent risk factors of myopia.

The prevalence of myopia among Chinese schoolchildren is often reported to be higher than children from other ethnic groups. The myopic rate was $1.4 \%$ in South America ${ }^{16}$ (children aged $5 \sim 15$ years) and $19.6 \%$ in France $^{17}$ (children aged $0 \sim 9$ years), Gryzbowski et al. summarized that the highest prevalence of myopia in children was in urban areas of China, Singapore and South Korea. ${ }^{18}$ The prevalence of myopia in Chongqing is growing rapidly over the recent 10 years. Pi et al. reported in 2010 the prevalence of myopia was $0.42 \% \sim 19.34 \%$ (children aged $6 \sim 12$ years) in suburban area of Chongqing. ${ }^{19}$ While according to Xie et al., the prevalence of myopia rose to $9.9 \% \sim 48.8 \%$ (children aged $7 \sim 12$ years) in $2020 .{ }^{20}$ Nevertheless, according to our study, myopic students who did not wear glasses accounted for $74.25 \%$ of the whole myopic population. Without adequate correction of refractive error, those students are at risk of having more challenges in their academic life and a more rapid progression of myopia. Those evidences showed that myopia in schoolchildren has become a major health concern in China. More measures should be taken to slow the progression of myopia and raise the rate of correction in myopic students.

Our study found that AL/CRC ratio was more highly correlated than AL alone with SER (AL: $r=-0.531$ vs. $A L / C R C: r=-0.639)$. Although greater AL was associated with a greater likelihood of myopia, myopic refractions were also found in shorter eyes. The finding of this research is consistent with other population-based studies of ocular biometry in children. Ip et al. reported that in Australian population, $\mathrm{AL} / \mathrm{CRC}$ ratio correlated better with refraction than $\mathrm{AL}$ alone. ${ }^{21}$ Scheiman et al. stated a larger correlation between AL/CRC and SER as myopia progressed in a longitudinal study in the United States. ${ }^{9} \mathrm{He}$ et al. stated that AL/CRC ratio could explain $65.7 \%$ of the variance in SER. ${ }^{22}$ Previous studies suggested that $\mathrm{AL} / \mathrm{CRC}$ could function as myopia detection indicator ${ }^{23}$ and considered an $\mathrm{AL} / \mathrm{CRC}$ ratio equal to or higher than 3 to be indicative of myopia. ${ }^{22,24,25}$ The current study found that the average AL/CRC among myopic subjects was 3.08 , and that among non-myopic subjects was $2.94 \pm 0.08$, similar to the findings from previous researches. ${ }^{25}$

Hyperopia reserve is also a potential indicator of myopia. At birth neonates display a wide range of refractions. There is a progressive shift in mean refraction from $+2 D$ to approximately $+0.75 D$. Then 
emmetropization continues at a slower rate after 6 years of age. Studies reported that the mean refraction is hyperopic in children at age of $6 \sim 7$ years from Australia and European countries, ${ }^{26}$ while it is the lowest in Japan. ${ }^{27}$ And Japan displays much higher rates of myopia in older children. So far there is no longitudinal study investigating the impact of low hyperopia reserve on future myopia development. In our study, the percentage of children with adequate hyperopia reserve was only $6.10 \%$ at age of $6 \sim 7$ years. We estimate that their incidence of myopia could be greater than their seniors. However, follow-up studies are required to validate its predictability.

Our results highlight that the significant association between the height, weight and BMI with AL, SER and the AL/CRC ratio. However, after adjusted by sex, age, parental myopia, and other risk factors, BMI could be associated with longer $A L(p=0.044)$. But BMI did not raise the risk of myopia. The current results are consistent with Ye et al., ${ }^{14}$ who demonstrated that among 482 Chinese children aged between $6 \sim 15$ years, taller individuals were associated with longer ALs $(b=+0.25, p<0.01)$, deeper VCDs $(b=+0.23$, $\mathrm{p}<0.01)$, higher $\mathrm{AL} / \mathrm{CC}$ ratios $(b=+0.04, \mathrm{p}<0.01)$ and more negative refractions $(b=-0.48, \mathrm{p}<0.01)$. Nonetheless, BMI was not correlated with refraction in their multiple linear regression models. A research from Singapore analyzing the relationship between $\mathrm{BMI}$ and myopia presented showed that $\mathrm{BMI}$ is associated with longer axial length in girls but not in boys. ${ }^{28}$ The relationship between $\mathrm{BMI}$ and refraction is not conclusive. An epidemiological study of myopia in Korea reported no relationship between body stature and myopia. ${ }^{29}$

Our observation of a strong association between parental myopia and myopia in children is consistent with previous findings. The risk of developing myopia was higher when both parents had myopia than only one parent. Jiang et al. suggested that parental myopia might contribute to a child's myopia by setting up a more myopic baseline at school age. ${ }^{30}$ The debate over the relative contribution of genetic vs environmental influences on myopia always exists. However, the importance of both is becoming more recognized.

Our study evaluated potential risk factors such as near visual work, electronic screen use, cram schools, reading posture and duration of outdoor activities. And we found that time of homework, near visual work, reading and writing posture, time of outdoor activities were not related with incidence of myopia. In previous studies, the impact of near visual work and time of homework on myopia formation was not consistent. According to the systematic review by Huang et al., longitudinal studies of incident myopia conducted in Australia, Taiwan, Singapore, and the United States reported that near work was not a significant risk factor for myopia development. ${ }^{31}$ Outdoor time was a significant protective factor for myopia in clinical trials and in cross-sectional studies. ${ }^{32}$ It is inconsistent with the current study, which might be owing to the rather low response rate of questionnaires. Cram schools are prevalent in China because of the heavy educational pressure. Many parents are keen on enhancement of children's academic performance. Time for outdoor activities is taken up by extra homework and examinations. Ku et al. reported that cram school attendance for more than $2 \mathrm{~h} /$ day may increase the risk of incident 
myopia in children from Taiwan. ${ }^{33}$ Our study found that time of cram schools are independently associated with increase of $A L$ and $A L / C R C$.

The strength of our study includes the large sample size with representative ocular biometric characteristics of schoolchildren in all grades. We demonstrated the importance of AL/CRC ratio as indicator of myopia. And we reported that hyperopia reserve could be a potential predictor of myopia in younger schoolchildren. The limitations of this study should also be discussed. We used social media mobile phone application as the platform to deliver and collect our questionnaire. The response rate is rather low, which limited our sample size when analyzing the risk factors of myopia. It might also cause a bias in our study because the parents who answered the questionnaire were possibly more attentive to the health care of their children.

\section{Conclusions}

This study found that in primary schools in Chongqing, the prevalence of myopia is alarming high. The rate of myopic students wearing spectacles is far from satisfying. Although myopia is still uncommon in children aged between $6 \sim 7$ years, the hyperopia reserve among them is low and they might be at a greater risk of developing myopia in the next few years. Outdoor activity is a protective factor against myopia. Health care providers need to raise parents' awareness of the importance of regular eye examination and proper optical correction. Children should be encouraged to walk out of their classroom during breaks between classes. Future longitudinal studies in the same population are planned to evaluate the predicative value of hyperopia reserve and other potential factors.

\section{Declarations}

Ethics approval and consent to participate

All of the study protocols conformed to the Tenets of the Declaration of Helsinki and the research protocols were approved by the Committee of Ethics in Chongqing People's Hospital. Written informed consent was obtained from a parent or guardian for all participants under 16 years old. Ethical approval number: KYS2021-011-01.

Consent for publication

All authors have made the consent for publication.

Availability of data and material

The data have not been placed in any online data storage. The datasets generated and analyzed during the study are available upon request from the first author.

Competing interests 
I declare that the authors have no competing interests as defined by $\mathrm{BMC}$, or other interests that might be perceived to influence the results and/or discussion reported in this paper.

\section{Funding}

This work is not supported by any funding.

Authors' contributions

Hong Li and Hong Yi contributed to the conception of the study. Yu Yue, Xianmao Liu, Bo Liu and Shu Yi contributed significantly to the data analysis and manuscript preparation;

Yu Yue and Xianmao Liu wrote the manuscript. Hong Li helped perform the analysis with constructive discussions. All authors have read and approved the manuscript.

Acknowledgements

We gave our heartfelt thanks to Jun Zhao, Changqin Zhu, Qingshu Cai and all the staff who participated in this epidemiological study for their hard work in the fieldwork. None of the authors has a financial or proprietary interest in any material or method mentioned.

\section{References}

1. Lyu Y, Zhang H, Gong Y, et al. Prevalence of and factors associated with myopia in primary school students in the Chaoyang District of Beijing, China. Japanese Journal of Ophthalmology, 2015;59:421-9.

2. Yam JC, Tang SM, Kam KW, et al. High prevalence of myopia in children and their parents in Hong Kong Chinese Population: the Hong Kong Children Eye Study. Acta Ophthalmol, 2020.

3. Ding B, Shih Y, Lin L, et al. Myopia among schoolchildren in East Asia and Singapore. Survey of ophthalmology, 2017;62:677-697.

4. Dong L, Kang YK, Li Y, et al. Prevalence and time trends of myopia in children and adolescents in China: A Systemic Review and Meta-Analysis. Retina, 2020;40:399-411.

5. Haarman A, Enthoven C, Tideman J, et al. The Complications of Myopia: A Review and MetaAnalysis. Investigative Ophthalmology \& Visual Science, 2020;61:49.

6. Ruiz-Medrano J, Montero JA, Flores-Moreno I, et al. Myopic maculopathy: Current status and proposal for a new classification and grading system (ATN). Progress in retinal and eye research, 2019;69:80-115.

7. Huang L, Kawasaki H, Liu Y, et al. The prevalence of myopia and the factors associated with it among university students in Nanjing: A cross-sectional study. Medicine, 2019;98:e14777.

8. Sun J, Zhou J, Zhao P, et al. High prevalence of myopia and high myopia in 5060 Chinese university students in Shanghai. Invest Ophthalmol Vis Sci, 2012;53:7504-9. 
9. Scheiman M, Gwiazda J, Zhang Q, et al. Longitudinal changes in corneal curvature and its relationship to axial length in the Correction of Myopia Evaluation Trial (COMET) cohort. Journal of optometry, 2016;9:13-21.

10. Flitcroft D. Emmetropisation and the aetiology of refractive errors. Eye (London, England), 2014;28:169-79.

11. Morgan IG, Rose KA. Myopia: is the nature-nurture debate finally over? Clinical and Experimental Optometry, 2019;102:3-17.

12. Morgan IG, French AN, Ashby RS, et al. The epidemics of myopia: Aetiology and prevention. Progress in retinal and eye research, 2018;62:134-149.

13. Wang J, Li M, Zhu D, et al. Smartphone Overuse and Visual Impairment in Children and Young Adults: Systematic Review and Meta-Analysis. Journal of medical Internet research, 2020;22:e21923.

14. Ye S, Liu S, Li W, et al. Associations between anthropometric indicators and both refraction and ocular biometrics in a cross-sectional study of Chinese schoolchildren. BMJ open, 2019;9:e027212e027212.

15. Wang N, Li S. The handbook for prevention and control of myopia in children and adolescents by the

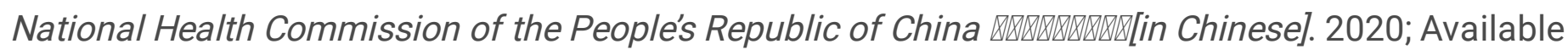
from: http://www.nhc.gov.cn/cms-search/downFiles/4198909572eb48ca9f3baab84b003f4d.pdf. Accessed August 24, 2021

16. Carter MJ, Lansingh VC, Schacht G, et al. Visual acuity and refraction by age for children of three different ethnic groups in Paraguay. Arq Bras Oftalmol, 2013;76:94-7.

17. Matamoros E, Ingrand P, Pelen F, et al. Prevalence of Myopia in France: A Cross-Sectional Analysis. Medicine, 2015;94:e1976.

18. Grzybowski A, Kanclerz P, Tsubota K, et al. A review on the epidemiology of myopia in school children worldwide. Bmc Ophthalmology, 2020;20:27.

19. Pi L, Chen L, Liu Q, et al. Refractive status and prevalence of refractive errors in suburban school-age children. International Journal of Medical Sciences, 2010;7:342-53.

20. Xie Z, Long Y, Wang J, et al. Prevalence of myopia and associated risk factors among primary students in Chongqing: multilevel modeling. BMC Ophthalmol, 2020;20:146.

21. Ip JM, Huynh SC, Kifley A, et al. Variation of the contribution from axial length and other oculometric parameters to refraction by age and ethnicity. Investigative Ophthalmology \& Visual Science, 2007;48:4846-53.

22. He X, Zou H, Lu L, et al. Axial length/corneal radius ratio: association with refractive state and role on myopia detection combined with visual acuity in Chinese schoolchildren. PLoS One, 2015;10:e0111766.

23. Wang SK, Guo Y, Liao C, et al. Incidence of and Factors Associated With Myopia and High Myopia in Chinese Children, Based on Refraction Without Cycloplegia. JAMA ophthalmology, 2018;136:10171024. 
24. Grosvenor T, Scott R. Role of the axial length/corneal radius ratio in determining the refractive state of the eye. Optometry and vision science : official publication of the American Academy of Optometry, 1994;71:573-9.

25. Guo X, Fu M, Ding X, et al. Significant Axial Elongation with Minimal Change in Refraction in 3- to 6Year-Old Chinese Preschoolers: The Shenzhen Kindergarten Eye Study. Ophthalmology, 2017;124:1826-1838.

26. French A, O'donoghue L, Morgan I, et al. Comparison of refraction and ocular biometry in European Caucasian children living in Northern Ireland and Sydney, Australia. Investigative Ophthalmology \& Visual Science, 2012;53:4021-31.

27. Watanabe S, Yamashita T, Ohba N. A longitudinal study of cycloplegic refraction in a cohort of 350 Japanese schoolchildren. Cycloplegic refraction. Ophthalmic Physiol Opt, 1999;19:22-9.

28. Saw S, Chua W, Hong C, et al. Height and its relationship to refraction and biometry parameters in Singapore Chinese children. Investigative Ophthalmology \& Visual Science, 2002;43:1408-13.

29. Jung S, Lee J, Kakizaki $\mathrm{H}$, et al. Prevalence of myopia and its association with body stature and educational level in 19-year-old male conscripts in seoul, South Korea. Investigative Ophthalmology \& Visual Science, 2012;53:5579-83.

30. Jiang X, Tarczy-Hornoch K, Cotter SA, et al. Association of Parental Myopia With Higher Risk of Myopia Among Multiethnic Children Before School Age. JAMA ophthalmology, 2020.

31. Huang H, Chang D, Wu P. The Association between Near Work Activities and Myopia in Children-A Systematic Review and Meta-Analysis. PLoS One, 2015;10:e0140419.

32. Xiong S, Sankaridurg P, Naduvilath T, et al. Time spent in outdoor activities in relation to myopia prevention and control: a meta-analysis and systematic review. Acta Ophthalmol, 2017;95:551-566.

33. Ku P, Steptoe A, Lai Y, et al. The Associations between Near Visual Activity and Incident Myopia in Children: A Nationwide 4-Year Follow-up Study. Ophthalmology, 2019;126:214-220.

\section{Figures}


SER

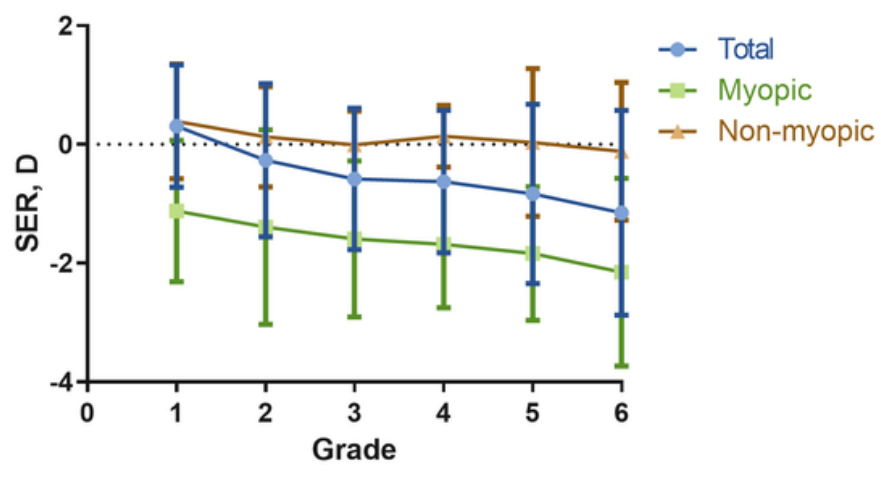

AL

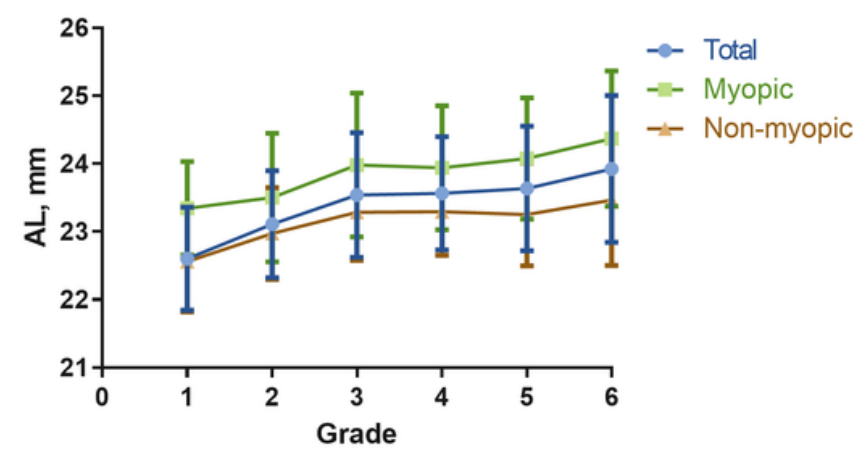

AL/CRC

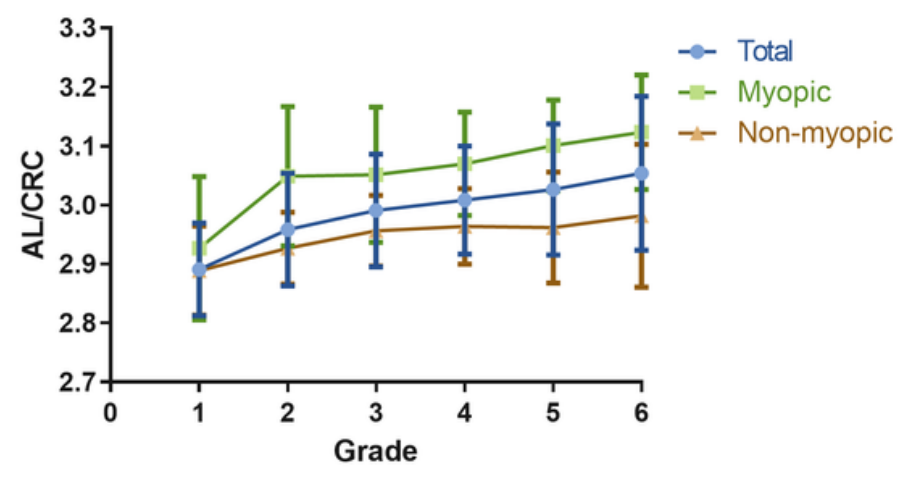

CRC

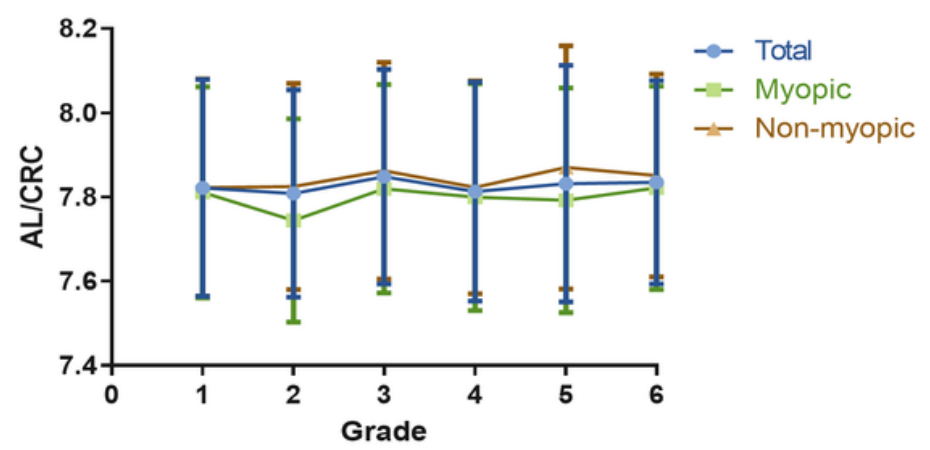

Figure 1

Dots represents means and bars represents SDs. SER: spherical equivalent refraction; AL: axial length; $\mathrm{CRC}$ : corneal radius of curvature; $\mathrm{AL} / \mathrm{CRC}$ : ratio of $\mathrm{AL}$ to $\mathrm{CRC}$. 


\section{Hyperopia Reserve}

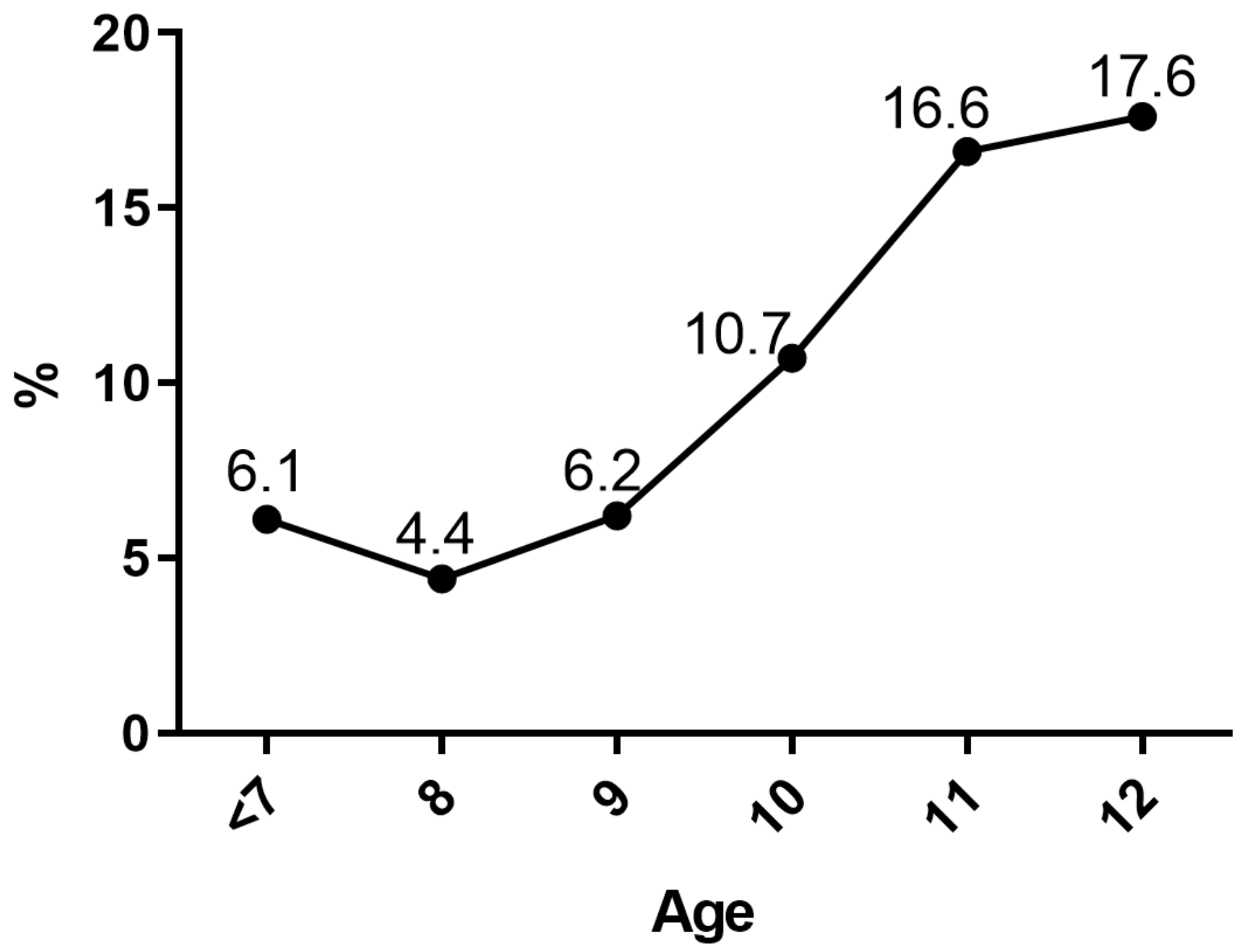

Figure 2

Rate of adequate hyperopia reserve among children aged less than 13 years old.

\section{Supplementary Files}

This is a list of supplementary files associated with this preprint. Click to download.

- S1questionnaireinEnglishd1.doc

- S2questionnaireinChinesed1.doc 
- S3univariateanalysisd1.doc

Page 19/19 